\title{
Polarographische Messungen der Atmung bei niedrigen Sauerstoffdrucken
}

\author{
D. Hegner und H. Glossmann \\ Institut für Pharmakologie und Toxikologie an der Veterinär-Medizinischen Fakultät \\ der Justus-Liebig-Universität Gießen (Direktor: Prof. Dr. med. M. Frimmer)
}

(Z. Naturforschg. 20 b, $234-244$ [1965] ; eingegangen am 12. November 1964)

\begin{abstract}
Die von LüввERs ${ }^{1}$ entwickelte polarographische Meßanordnung wurde für die Registrierung der Atmung an isolierten Zellen und Mitochondrien bei niedrigen $\mathrm{O}_{2}$-Drucken adaptiert. Bei dieser Versuchsanordnung verbrauchen die atmenden Partikel den im System gelösten Sauerstoff, so daß der $P_{\mathrm{O}_{2}}$ laufend absinkt. Die Geschwindigkeit des $\mathrm{O}_{2}$-Verbrauches bleibt in einem weiten Bereich konstant und wird erst von einem kritischen $\mathrm{O}_{2}$-Druck (KD) an vermindert. Als Halbwertdruck (HWD) wird der $P_{\mathrm{O}_{2}}$ bezeichnet, bei dem die Atmung noch $50 \%$ des maximalen Umsatzes beträgt.

Bei Herzmuskel-Sarkosomen, Leber-Mitochondrien, Tumorzell-Mitochondrien und Ascites-Tumorzellen bleibt der KD und HWD innerhalb eines bestimmten Bereiches konstant, wenn man im Versuchsansatz die Konzentration der atmenden Partikel steigert. Von einer bestimmten Konzentration bzw. Atmungsgröße an (Grenzmaximalatmung), die temperaturabhängig ist, steigt zuerst der HWD und dann der KD in Abhängigkeit von der Konzentration der atmenden Partikel. Im Bereich jenseits der Grenzmaximalatmung ist die polarographische Meßanordnung nicht mehr zuverlässig, da dort eine zunehmende Aggregation der Partikel stattfindet.

Unterhalb der Grenzmaximalatmung ist der HWD dem $Q_{0_{2}} / \mathrm{mg}$ Protein der atmenden Partikel direkt, dem KD umgekehrt proportional. Der KD nimmt bei steigender Temperatur und damit zunehmender Maximalatmung ab. Berücksichtigt man die Grenzmaximalatmung und damit die Grenze der Zuverlässigkeit der polarographischen Methode, so findet man für $37^{\circ} \mathrm{C}$ niedrigere KD's als sie bisher angegeben wurden.

Bei niedrigen $P_{\mathrm{O}_{2}}$ ist $\frac{v_{\mathrm{max}}-v}{v}$ von $P_{\mathrm{o} 2}^{r}$ abhängig. Bei $22,5^{\circ} \mathrm{C}$ ist,$r^{\prime \prime} \operatorname{dem} Q_{\mathrm{O}_{2}} / \mathrm{mg}$ Protein proportional. Integriert man die $\mathrm{M}$ i c h a e l is - Funktion für , $r=$ verschieden von 1", so stimmen die errechneten und gemessenen Punkte der Atmungskurven überein, sofern die Grenzmaximalatmung nicht überschritten wird.
\end{abstract}

Die Atmung von Mitochondrien ist in einem weiten Konzentrationsbereich vom Sauerstoffdruck unabhängig. Sie nimmt erst bei verhältnismäßig niederen $\mathrm{O}_{2}$-Partialdrucken (Kritischer Druck) ab. Die Größe des kritischen $\mathrm{O}_{2}$-Druckes interessiert im $\mathrm{Zu}$ sammenhang zahlreicher pathophysiologischer Probleme der Hypoxie und Anoxie. Die Messung kritischer Drucke und sog. Halbwertdrucke * kann auf zweierlei Weise erfolgen:

1. Bei der manometrischen Messung des $\mathrm{O}_{2}$-Verbrauchs isolierter Zellen oder Mitochondrien wird im Bereich niederer $\mathrm{O}_{2}$-Drucke die Diffusion vom Gasraum in die Suspension der atmenden Partikel limitierend. Durch rasche Rotation des Meßgefäßes auf einem Exzenter gelang es Warburg und Kubo$\mathrm{W}_{\mathrm{ITZ}}{ }^{2}$ sowie BäNDER und $\mathrm{KIESE}^{3}$ die klassische manometrische Methode so zu modifizieren, daß die Oberfläche der Suspension genügend groß wurde

1 U. Gleichmann u. D. W. Lübbers unter technischer Mitwirkung von W. Bürger u. W. Eschweiler, Pflügers Arch. ges. Physiol. Menschen Tiere 271, 431 [1960].

* $\mathrm{O}_{2}$-Druck, bei dem Atmung halb so groß ist wie unter Luft. und die Diffusion zu vernachlässigen war. Die Nachteile des Verfahrens liegen in der Gefahr der Beschädigung der Mitochondrien, in der Aufwendigkeit der Apparatur und in der Unmöglichkeit der Durchführung größerer Meßserien mit einer Mitochondrien-Präparation.

2. Bei der polarographischen Messung des $\mathrm{O}_{2}$ Verbrauchs veratmen suspendierte Mitochondrien den physikalisch gelösten Sauerstoff des Suspensionsmediums. Die Versuchsanordnung enthält keinen Gasraum. Deswegen entfällt das Problem der Diffusion vom Gasraum zum Suspensionsmedium. Da durch den $\mathrm{O}_{2}$-Verbrauch die $\mathrm{O}_{2}$-Spannung der Suspensionsflüssigkeit laufend absinkt, kann in jeweils einem Versuch die Abhängigkeit der Reaktionsgeschwindigkeit von der $\mathrm{O}_{2}$-Konzentration direkt gemessen werden. Voraussetzung ist dabei, daß die Substratkonzentration genügend hoch ist. Dieses

2 O. Warburg u. F. Kubowitz, Biochem. Z. 241, 5 [1929].

3 A. B̈̈nder u. M. Kiese, Arch. exp. Path. u. Pharmak. 224, 312 [1955]. 
Verfahren erlaubt, in kurzer Zeit viele Messungen durchzuführen.

Die Vorteile der polarographischen Meßtechnik wurden von TöDT ${ }^{4}$ ausführlich diskutiert. Polarographische Messungen wurden bereits 1941 von Winzler $^{5}$ an Bäckerhefe mit der Hg-Tropfelektrode, 1954 und 1957 von LoNGmuir ${ }^{6,7}$ an Bakterien und Rattenleberzellen mit der Goldelektrode, von $\mathrm{Chance}^{8}$ und David C. $\mathrm{White}^{9}$ mit einer Platinelektrode durchgeführt.

Die von Lübbers und Mitarbb. ${ }^{1}$ zur Messung von $\mathrm{O}_{2}$-Spannungen im Blut entwickelte Ganzglas-Platinelektrode versetzte uns in die Lage, ohne nennenswerte Schwierigkeiten die Abhängigkeit des $\mathrm{O}_{2}$-Verbrauchs von der $\mathrm{O}_{2}$-Konzentration bei Leber-Mitochondrien, Herzmuskel-Sarkomen, Ascites-Tumorzellen und Tumorzell-Mitochondrien unter verschiedenen experimentellen Bedingungen zu messen. Ziel dieser Arbeit war, das für die Messung stationärer, verhältnismäßig hoher $\mathrm{O}_{2}$-Konzentrationen entwikkelte Verfahren für die Registrierung des $\mathrm{O}_{2}$-Verbrauchs bei extrem niedrigen Drucken zu adaptieren. Bei früheren Untersuchungen ${ }^{10}$ war uns aufgefallen, daß die Mitochondrienmenge im Versuchsansatz nicht ohne Einfluß auf die gemessenen Halbwert- und kritischen Drucke der $\mathrm{O}_{2}$-abhängigen Atmung war, sofern die maximale $\mathrm{O}_{2}$-Aufnahme einen bestimmten Höchstwert überschritt. Wir haben versucht, dieses Phänomen für die von uns verwendete Meßanordnung zu klären. Da die in der Literatur bereits mitgeteilten Daten von Halbwertdrucken, Kritischen Drucken bei Mitochondrien und Mikroorganismen erheblich voneinander abweichen und auch die Deutung der Befunde uneinheitlich ist, haben wir das Problem erneut bearbeitet.

$\mathrm{W}_{\text {ARBURg und KuBowitz }}{ }^{2}$ fanden, daß bei Kugelbakterien die kritischen Drucke um so niedriger waren, je kleiner die verwandte Zellmenge oder je größer das verwandte Suspensionsvolumen war. Als Ursache hier-für sahen diese Verfasser die Begrenzung des Gasaustausches zwischen Gasraum und Flüssigkeit an. Bei tieferen Temperaturen waren die kritischen Drucke niedriger, was mit der größeren Löslichkeit des Sauerstoffes erklärt wurde.

${ }^{4}$ F. TöDt, Walter Gruyter-Verlag, Berlin 1958.

5 R. J. Winzler, J. cellular comparat. Physiol. 17, 263 [1941].

6 I. S. Longmuir, Biochem. J. 57, 81 [1954].

7 I. S. Longmuir, Biochem. J. 65, 378 [1957].

8 B. Chance, Nature [London] 169, 215 [1952].

9 C. David White, J. biol. Chemistry 238, 3757 [1963].

10 M. Frimmer, D. Hegner u. W. Winkelmann, Klin. W schr. 41, 715 [1963].
$\mathrm{W}_{\text {INZLeR }}{ }^{5}$ fand bei Bäckerhefe ebenfalls eine $\mathrm{Ab}$ hängigkeit des kritischen Druckes von der Temperatur $\left(0,6\right.$ Torr bei $5{ }^{\circ} \mathrm{C}, 6$ Torr bei $\left.34{ }^{\circ} \mathrm{C}\right)$. Weder die gröBere Löslichkeit des Sauerstoffes bei niedrigen Temperaturen, noch Diffusion durch das Zellprotoplasma, sondern eine Eigenschaft des $\mathrm{O}_{2}$-übertragenden Fermentes ist nach $\mathbb{W}_{\text {INZLER }}$ die Ursache für dieses Phänomen. BäNDER und KIESE ${ }^{3}$ konnten aus methodischen Gründen den kritischen $\mathrm{O}_{2}$-Druck für Rattenleber-Mitochondrien nur näherungsweise bestimmen. Longmuir ${ }^{6}$ fand im Gegensatz zu den oben erwähnten Untersuchern einen "wahren kritischen Druck", d.h. bei einem ganz bestimmten $\mathrm{O}_{2}$-Druck fiel bei seiner Versuchsanordnung die Geschwindigkeit der Atmung plötzlich ab. Dieser „wahre kritische Druck“ war nach Longmuir um so besser meßbar, je größer die Maximalgeschwindigkeit gewählt wurde. LoNGMuir ${ }^{6}$ gab aber für diesen „wahren kritischen Druck" keinen Zahlenwert an. Er wies lediglich daraufhin, daß er etwa 4-5-mal so groß wie der Halbwertdruck sei.

Ähnliche divergierende Ergebnisse und Deutungen finden sich in der Literatur über den Halbwertdruck. W $_{\text {ARBurg }}$ und Kubowitz fanden bei Kugelbakterien und $\mathrm{W}_{\text {InzLER }}{ }^{5}$ bei Bäckerhefe eine Abhängigkeit des Halbwertdruckes (HWD) von der Temperatur. Er wurde mit steigender Temperatur größer - verhielt sich also ähnlich dem kritischen Druck. WINzLER wies für Bäckerhefe nach, daß die Diffusion durch das Plasma für die Größe des HWD nicht bestimmend sein kann. Im Gegensatz dazu fand Longmuir ${ }^{6}$ eine direkte Proportionalität zwischen HWD und Größe der von ihm untersuchten Organismen*.

Bei größeren Organismen spielt nach Longmuir ${ }^{6}$ die Diffusion eine entscheidende Rolle für die Größe des HWD. Das größte von ihm untersuchte Objekt, die Bäckerhefe (Zelldurchmesser etwa 5,5 $\mu$ ), bildete eine Ausnahme. Bei Hefe wurde merkwürdigerweise nicht der größte HWD ermittelt. 1957 untersuchte Longmuir ${ }^{7}$ Rattenleberzellen und fand eine Abhängigkeit des HWD von der Succinatkonzentration und der Temperatur. Die HWD's waren der Maximalgeschwindigkeit direkt proportional. Bei einem Vergleich der HWD's für Rattenleberzellen mit den von BäNDER und KIESE ${ }^{3}$ untersuchten Rattenleber-Mitochondrien wies Longmuir ${ }^{7}$ daraufhin (ohne Meßwerte anzugeben), daß die Diffusion des Sauerstoffes durch das Protoplasma kein limitierender Faktor sei. Aktivierungsenergie und Grundreaktionswärme seien für Zellen und Mitochondrien etwa gleich, während der HWD für Zellen bei vergleichbarer Atmungsgröße etwas höher liege.

11 B. Chance, Federat. Proc. 16, 671 [1957].

12 W. C. Schneider u. G. H. Hogeboom, A review, Cancer Res., II, I [1951].

13 A. L. Lehninger u. E. P. Kennedy, J. biol. Chemistry 123, 753 [1948].

* $K_{\mathrm{m}}=A \times d^{\mathrm{x}} \quad\left(K_{\mathrm{m}}=\mathrm{HWD}\right.$ in $\mathrm{Mol} / l \mathrm{H}_{2} \mathrm{O}, A=$ Proportionalitätsfaktor, $d=$ Durchmesser des Objektes, $x=$ Konstante). 
BäNDER und $\mathrm{KIESE}^{3}$ fanden, daß im Bereich von 10 bis $90 \%$ der Maximalatmung für Bäckerhefe ${ }^{5}$, für $M i$ crococcus cand. ${ }^{2}$ und für die von ihnen selbst untersuchten Rattenleber-Mitochondrien folgende Beziehung gilt :

$$
\frac{v}{v_{\max }-v}=\frac{K_{\text {red }}}{K_{0 \mathrm{x}}} P_{02}^{r} r=1,4 .
$$

Longmuir ${ }^{13}$ integrierte die $\mathrm{Mich}$ a elis-Funktion (für $r=1$ ) und fand bei Bakterien eine gute Über. einstimmung der berechneten Druck-Zeit-Kurven mit den experimentellen Ergebnissen. Für Rattenleberzellen bestätigte er $1957^{7} \mathrm{im}$ Bereich bis $80 \%$ bzw. $90 \%$ der Maximalatmung die von B̈̈NDER und $\mathrm{KIESE}^{3}$ gefundene Beziehung (1), allerdings mit der Einschränkung, daß oberhalb $80 \%$ der Maximalatmung der Exponent , ${ }^{\prime \prime}$ größer als 1,4 ist. Change ${ }^{8,11}$ hat Halbwertdrucke der $\mathrm{O}_{2}$-Aufnahme mit der Platinelektrode an einer Schweineherz-Präparation und Keilin-HartreePräparation gemessen (Tab. 1). Er weist darauf hin, daß die von Longmurr ${ }^{6}$ gefundenen, auffallend niedrigen Halbwertdrucke wahrscheinlich auf Inaktivierung der Präparationen zurückzuführen sind. Chance und Mitarb. ${ }^{11}$ haben den Exponenten „r" theoretisch berechnet und finden dafür einen Wert von 1,33. David C. White ${ }^{9}$ konnte zeigen, daß durch Änderung der

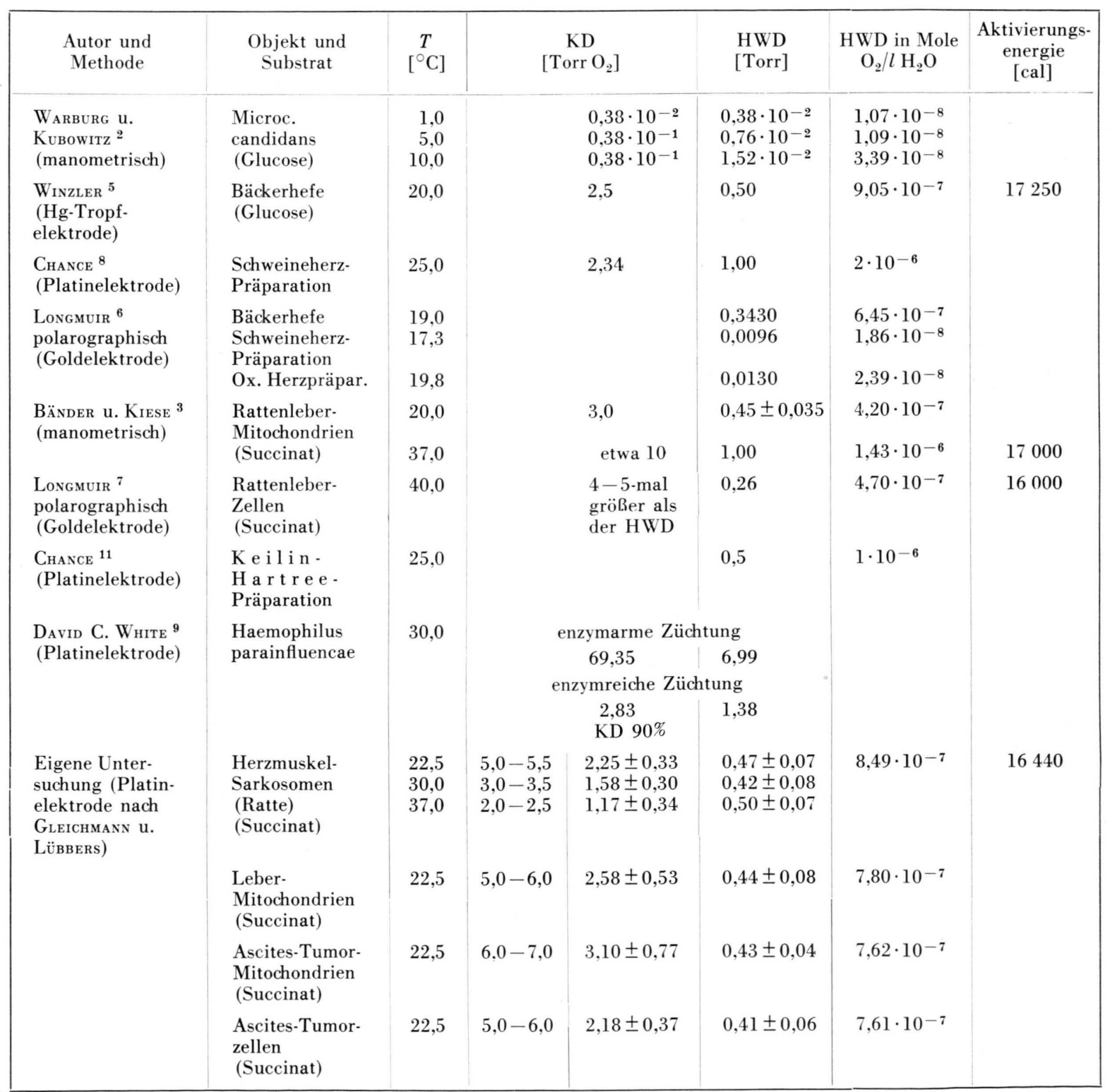

Tab. 1. Zusammenstellung von Messungen kritischer und Halbwertdrucke an verschiedenen Objekten. 
Enzymkonzentration (Cytochromoxydase) kritische und Halbwertdruck bei enzymarmen Präparationen höher waren als bei enzymreichen (Tab. 1).

Wir haben die Zell- bzw. die Mitochondrienmenge bei konstanter Temperatur und maximaler Sättigung des Systems mit Succinat variiert und damit die Maximalgeschwindigkeit der $\mathrm{O}_{2}$-Aufnahme verändert und HWD und KD (kritischer Druck) bestimmt. Außerdem wurde der Exponent " $r$ “ der Funktion (1) bestimmt. Die Mich a elis-Funktion wurde für die bei Sarkomen, Leber-Mitochondrien, AscitesTumorzellen und -Mitochondrien verschiedenen Exponenten " $r$ “ integriert und die Druck-Zeit-Kurven mit den so errechneten Werten verglichen. Der Exponent " $r$ " war für die verschiedenen atmenden Objekte abhängig von der Maximalatmung $\left(\mathrm{O}_{2}\right.$-Aufnahme pro mg Protein). Die Halbwertdrucke nahmen für verschiedene Objekte ebenso wie " $r$ “ mit zunehmenden $Q_{\mathrm{O}_{2}} \mathrm{zu}$. Dagegen waren die KD's dem $Q_{\mathrm{O}_{2}}$ umgekehrt proportional und nahmen mit zunehmender Temperatur ab.

\section{Methode}

Rattenleber-Mitochondrien wurden in der üblichen Weise nach Schneider ${ }^{12}$, Lehninger und Kennedy ${ }^{13}$ präpariert. Die Mitochondrien wurden zum Schluß $4-5$-mal in 0,25-m. Sucroselösung auf der Kühlzentrifuge gewaschen und anschließend in SVT $(0,25-m$. Sucrose, $0,05-m$. TRIS, $0,1 \mathrm{mMol}$ EDTA, $p_{\mathrm{H}}$ 7,4) suspendiert.

Sarkosomen wurden aus Herzen von $200-250 \mathrm{~g}$ schweren Wistarratten gewonnen. Für eine Präparation wurden 5-6 Tiere durch Entbluten getötet. Die Herzen wurden sofort nach Entnahme in $50 \mathrm{ml}$ einer eiskalten Lösung $1(0,21-m$. Mannit und 0,07-m. Sucrose) eingebracht. Alle Arbeitsgänge wurden bei $0{ }^{\circ} \mathrm{C}$ durchgeführt. Die Herzen wurden gewogen, mit der Schere zerkleinert und je $6 \mathrm{~g}$ Herzmuskel in eine Lösung gegeben, die aus $100 \mathrm{ml}$ Lösung 1, $60 \mathrm{mg}$ Nargase (Subtilisin A, krist. reinst der Fa. Serva) und 2,2 ml TRAPuffer $\left(0,5-m . p_{\mathrm{H}} 7,6\right)$ bestand. Nach 45 Min. Einwirkungsdauer des Enzyms wurde im Potter-Homogenisator vorsichtig homogenisiert. Nach weiteren 20 Min. wurden $100 \mathrm{ml}$ Lösung 2 zugegeben (0,21-m. Mannit, 0,07-m. Saccharose und $0,1 \mathrm{mMol}$ EDTA) und erneut homogenisiert. Anschließend wurde 2-mal zentrifugiert (4700 $\mathrm{g}$ während 5 Min.). Der Rückstand wurde jeweils verworfen. Der Überstand wurde abgegeossen und $7 \mathrm{Min}$. bei $12300 \mathrm{~g}$ zentrifugiert. Der Niederschlag wurde in $10 \mathrm{ml} \mathrm{1-proz.} \mathrm{Albuminlösung} \mathrm{(Serumalbumin}$ vom Rind, reinst, gelöst in Lösung 2) aufgenommen und erneut $7 \mathrm{Min}$. bei $12300 \mathrm{~g}$ zentrifugiert. Der letzte Arbeitsgang wurde 4-5-mal wiederholt, bis das Sediment weitgehend frei von Erythrozyten, Hämoglobin und endogenem Substrat war. Zum Schluß wurden die
Sarkosomen in Lösung 1 aufgenommen und im Handhomogenisator vorsichtig aufgewirbelt.

Mitochondrien von Ehrlich-Ascites-Tumorzellen wurden nach dem von $\mathrm{H}_{\text {Awtrey }}$ und $\mathrm{S}_{\mathrm{ILK}}{ }^{14}$ angegebenen Verfahren präpariert, 3-mal gewaschen und in SV'T suspendiert.

Die in dieser Weise präparierten Mitochondrien und Sarkomen waren bei mikroskopischer Kontrolle frei von cytoplasmatischem Material. Die $\mathrm{O}_{2}$-Aufnahme dieser Mitochondrien war ohne Zusatz von Substrat praktisch zu vernachlässigen. In diesen Mitochondrien waren Atmung und Phosphorylierung bis zu 4 Stdn. nach der Präparation noch gut gekoppelt.

Ehrlich-Ascites-Tumorzellen wurden 10-14 Tage nach der Inokulation entnommen und in Tyrodelösung mit Citratzusatz 4-5-mal auf der Zentrifuge gewaschen $(1000 \mathrm{~g})$, anschließend in TRIS-Tyrode-Lösung $\left(p_{\mathrm{H}} 7,4\right)$ suspendiert. Erythrozyten-Beimengungen wurden durch Sedimentieren entfernt.

In Anlehnung an die Versuche von BäNDER und $\mathrm{KIESE}^{3}$ wurden die Bedingungen für eine maximale $\mathrm{O}_{2}$-Aufnahme der Präparationen so gewählt, daß Succinat als Wasserstoffdonator diente. Im Versuchsansatz waren: $0,5 \mathrm{ml} 0,024-m$. TRIS-Puffer, $p_{\mathrm{H}} 7,4,0,25 \mathrm{ml}$ 0,025-m. $\mathrm{MgSO}_{4}, 0,5 \mathrm{ml} 3,5 \cdot 10^{-4}-m$. Cytochrom c, $0,25 \mathrm{ml}$ 5-proz. Serumalbumin, $0,25 \mathrm{ml} 0,64-\mathrm{m}$. NaSuccinat und Mitochondrien- bzw. Zellsuspensionen. Die Mitochondrien- bzw. Zellmenge wurde so variiert, daß die maximale $\mathrm{O}_{2}$-Aufnahme (bei Luftsättigung) 0,02 bis $0,55 \mu \mathrm{l} \mathrm{O} / \mathrm{ml} \mathrm{Ansatz} / \mathrm{Min}$. betrug. Es wurde soviel Suspensionsmedium zugegeben, daß die Gesamtmenge des Versuchsansatzes 2,0 bzw. $2,5 \mathrm{ml}$ betrug.

Bei einigen Versuchen wurde die Succinatkonzentration auf 0,14-m. verringert. Atmung und Halbwertdrucke waren bei $0,64-m$. und $0,14-m$. Na-Succinat gleich.

Zur Stabilisierung der Mitochondrien und Zellen verwandten wir reines Rinderserumalbumin. Ungereinigte Albuminpräparate enthalten Spuren von Substraten, die im Versuchsansatz mit Phosphatpupuffer ohne Succinat eine „endogene“ Atmung vortäuschten. Deshalb verwandten wir TRIS-Puffer und Serumalbumin "reinst“ (Behring-Werke), Cytochrom c (nach K e i $\mathrm{l}$ in und $\mathrm{Ha} \mathrm{rtree}$, salzfrei) von der Fa. Boehringer.

Die Mitochondrien-Konzentration wurde durch Bestimmung des Proteingehaltes nach der Biuret-Methode ermittelt. Ascitestumorzellen wurden in einer Zählkammer nach Thom a s ausgezählt (Zahl der Zellen je $\mathrm{mm}^{3}$ Ansatz). Da das Alter der Mitochondrien die $\mathrm{O}_{2}$ Aufnahme beeinflußte, wurden frische Mitochondrien nur bis maximal 4 Stdn. nach der Präparation zur Messung verwendet. So konnten pro Präparation $15-20$ Einzelmessungen durchgeführt werden. „Alte“ Mitochondrien waren maximal $24 \mathrm{Stdn}$. alt.

\section{Versuchsanordnung}

Der $\mathrm{O}_{2}$-Verbrauch der Ansätze wurde polarographisch mit einer membranbespannten Platinelektrode fortlaufend gemessen. Wir benutzten die von LübBERS 14 A. O. Hawtree u. M. Silk, Biochem. J. 74, 21 [1960]. 
und Mitarb. ${ }^{1}$ entwickelte stabilisierte Ganzglasplatinelektrode. Die Platinoberfläche hatte einen Durchmesser von 1,5 und $2,0 \mathrm{~mm}$ und wurde mit $12 \mu$ Cellophan und einer $25 \mu$ dicken Teflon-Membran bespannt. Die Prüfung und Eichung der Elektrode erfolgte nach Lübsers und Mitarb. ${ }^{1}$ angegebenen Verfahren. Mit einem von der Fa. Eschweiler und Co. gebauten Polarographen und einem Philips-Direktschreiber wurde fortlaufend die $\mathrm{O}_{2}$-Spannung registriert (Papiervorschub: $4 \mathrm{~cm}$ und $2 \mathrm{~cm} / \mathrm{Min}$.).

Der Versuchsansatz wurde luftblasenfrei in die Meßkammer gefüllt und von der umgebenen Atmosphäre durch Schließen von zwei Glashähnen getrennt. Kleinste Luftbläschen mußten unbedingt entfernt werden, da sonst Sauerstoff aus diesen Gasbläschen in die Meßlösung diffundierte und das Meßergebnis verfälschte.

Im Meßgefäß befand sich ein Magnetrührer (370 U/ Min.). Der Abstand des Rührers von der Elektrodenspitze mußte möglichst klein gehalten werden. Der Einfluß des Rührens auf die Stabilität der Mitochondrien wurde folgendermaßen geprüft: Die Mitochondrien des Versuchsansatzes verbrauchen allmählich den gesamten gelösten Sauerstoff. Setzt man nach Ablauf des Versuches den Ansatz erneut mit Luftsauerstoff ins Gleichgewicht und wiederholt dann die Messung, so bleibt die Anfangsgeschwindigkeit der $\mathrm{O}_{2}$-Aufnahme und der HWD bei den Versuchen gleich.

Um die mechanische Schädigung der Mitochondrien möglichst herabzusetzen, wurden die Ansätze unmittelbar vor der Messung mit Stickstoff auf $10-15$ Torr $\mathrm{O}_{2}$ äquilibriert. Die Meßdauer betrug dann selbst bei niedrigen Maximalgeschwindigkeiten nicht mehr als 20 Minuten. Die Temperaturkonstanz während der Messung war durch Eintauchen der Meßkammer in ein thermoreguliertes Wasserbad gewährleistet.

\section{Eichung der Ansätze}

Der Versuchsansatz wurde ohne Mitochondrien mit atmosphärischer Luft gesättigt. Im Meßgefäß wurde bei der jeweiligen Versuchstemperatur der Diffusionsgrenzstrom registriert. Dieser der $\mathrm{O}_{2}$-Spannung proportionale Strom wurde als „Ansatz-Eichwert“ bezeichnet. Alle registrierten Meßwerte wurden auf diese Eichung bezogen, die sich nur unwesentlich $(3-5 \%)$ von der Eichung mit Wasserdampf gesättigter Luft unterschied. Bei der Berechnung des $\mathrm{O}_{2}$-Gehaltes wurde der jeweilige Luftdruck und der Wasserdampfdruck berücksichtigt. Beim $\mathrm{O}_{2}$-Partialdruck der Luft (156 Torr) betrug die Stromstärke bei der Elektrode mit $2 \mathrm{~mm} \phi$ etwa 4,2 $\mu \mathrm{A}$ und bei der Elektrode mit 1,5 $\mathrm{mm} \phi$ etwa 2,4 $\mu$ A. Bei 20-facher Verstärkung entsprach der Vollausschlag des Ampèremeters etwa 8,5 Torr (Elektrode mit $2 \mathrm{~mm} \phi)$. Zur Zeigerberuhigung war in dem Gegenkopplungswiderstand ein Kondensator $(2500 \mathrm{pF})$ eingeschaltet. Kondensatoren mit größerer Kapazität bewirken zwar bessere Zeigerberuhigung, verzögern jedoch die Anzeige des $\mathrm{O}_{2}$-Diffusionsstromes und können das Meßergebnis verfälschen. (Nach $\mathrm{T}$ ö dt führen Kondensatoren von $100-1000 \mu \mathrm{F}$ zur Verzögerung der Anzeige um Sekunden.)

\section{Fehlerquellen der Methode \\ 1. $\mathrm{O}_{2}$-Eigenverbrauch der Elektrode}

War bei den von uns verwandten Elektroden die Stromstärke bei 156 Torr $\mathrm{O}_{2} 4,2 \mu \mathrm{A}(\phi 2 \mathrm{~mm}$, $22,5{ }^{\circ} \mathrm{C}$ ), so betrug der Eigenverbrauch (Volumen des Analysengefäßes $0,8 \mathrm{ml}$ ) im Bereich von 3 Torr 0,0013 Torr/Minute.

\section{Verschmutzung der Elektrodenoberfläche und Änderung der Diffusionsschicht}

Bei unseren Versuchen bewährte es sich, jeweils nach einigen Messungen von biologischem Material eine Kontrollmessung einzuschalten, während das Meßgefäß mit dest. Wasser gefüllt war. Der Diffusionsgrenzstrom wurde dann über einen Zeitraum von 5 Min. registriert. Während dieser Zeit durfte die Stromstärke nur um einen kleinen Betrag gemäß :

$$
i_{\mathrm{g}}=i_{\mathrm{g}_{0}} \cdot e \frac{-D \cdot q}{\delta \cdot V} \cdot t^{*}
$$

abnehmen.

Nimmt die Stromstärke stärker ab, so muß entweder der Abstand des Rührers zur Elektrode verkleinert oder die Elektrode erneuert werden.

\section{3. "Paradoxer" Strom}

In seltenen Fällen kann ein schwacher „paradoxer“ Strom fließen, dessen Stromrichtung der des Diffusionsgrenzstromes umgekehrt gerichtet ist. Die Registrierung gelangt dabei unter die O-Linie, wenn der $\mathrm{O}_{2}$-Partialdruck gleich null ist. Die Elektrode muß dann erneuert werden.

\section{Ergebnisse}

1. Abhängigkeit der maximalen $\mathrm{O}_{2}$-Aufnahme von der Mitochondrien-bzw. Zellkonzentration (Abb. 1)

Die Maximalgeschwindigkeit der $\mathrm{O}_{2}$-Aufnahme war bei allen untersuchten Objekten $\left(22,5^{\circ} \mathrm{C}\right)$ der Mitochondrien- bzw. Zellkonzentration proportional. Sie war bezogen auf mg Protein bei HerzmuskelSarkomen am größten und bei Ascites-Tumor-Mitochondrien am kleinsten.

\section{Die Atmung}

Sie war von etwa 156 Torr bis 6 Torr $\mathrm{O}_{2}$ bei $22,5{ }^{\circ} \mathrm{C}$ unabhängig vom $\mathrm{O}_{2}$-Druck. Für $30^{\circ}$ und $37^{\circ} \mathrm{C}$ gilt der Bereich der $\mathrm{PO}_{2}$-unabhängigen

* $D=$ Diffusionskoeffizient für $\mathrm{O}_{2}$ in $\mathrm{cm}^{2} /$ Sek., $q=$ Elektrodenoberfläche in $\mathrm{cm}^{2}, \delta=\mathrm{O}_{2}$-Diffusionsschicht in $\mathrm{cm}$, $V=$ Volumen des Analysengefäßes in $\mathrm{cm}$ (Näheres s. Töрт) . 


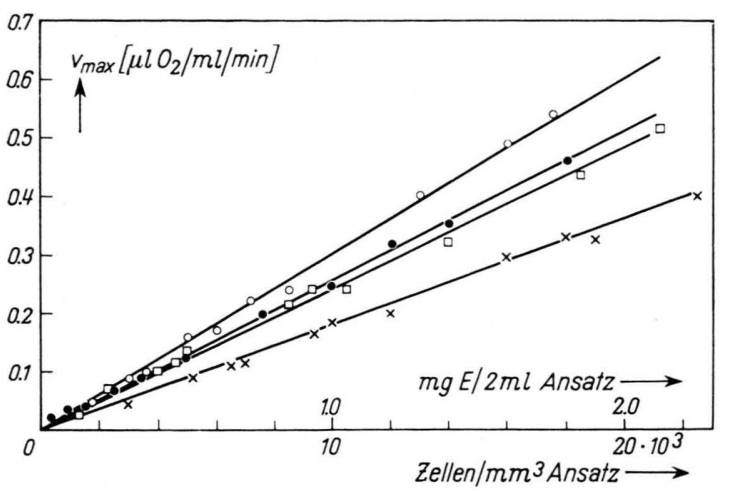

Abb. 1. Abhängigkeit der Maximalatmung von der Mitochondrien- und Zellkonzentration bei $22,5^{\circ} \mathrm{C}$. $\bigcirc=$ Herzmuskel-Sarkosomen, $\square=$ Rattenleber-Mitochondrien, $\quad \times=$ Ascites-Tumor-Mitochondrien, $\bullet=$ Ascites-Tumorzellen.

Atmung vom $\mathrm{PO}_{2}$ unter Luft bis 3 bzw. 2 Torr (innerhalb dieses Bereiches entspricht die Atmung einer Reaktion 0. Ordnung). Von 4 bis 6 Torr $\left(22,5{ }^{\circ} \mathrm{C}\right) 1.2$ nimmt die Geschwindigkeit bei allen untersuchten Objekten zunächst schneller ab, als es der Funktion (1) mit dem Exponenten " " " entsprechen würde. 0.8 Hier gilt ein Exponent,$s "$ der größer als,$r "$ ist ${ }_{0.6}$ (Abb. 3). Zwischen 4 und 6 Torr $\left(22,5{ }^{\circ} \mathrm{C}\right)$ liegt der kritische Druck (KD). Dieser war an unseren ${ }^{0.4}$ registrierten Kurven um so genauer zu bestimmen, 0.2 je geringer die Maximalgeschwindigkeit der $\mathrm{O}_{2}$-Aufnahme, d. h. je niedriger die Mitochondrien- bzw. Zellkonzentration pro ml Ansatz war. Bei hohen Maximalgeschwindigkeiten der Atmung konnten wir den KD nur näherungsweise bestimmen. Wir ermittelten daher für verschiedene Maximalgeschwindigkeiten der $\Theta_{2}$-Aufnahme am gleichen Objekt den genauer bestimmbaren KD 90 Prozent *. Die gefundenen Werte sind für die verschiedenen Objekte in Tab. 1 aufgeführt und stellen Mittelwerte aus jeweils 80 Messungen dar.

Dieser KD 90\% war von der Mitochondrien- bzw. Zellkonzentration bis zu einer Maximalatmung von $0,50 \mu \mathrm{l} \mathrm{O}_{2} / \mathrm{ml} / \mathrm{Min}$. bei gleicher Temperatur unabhängig, wenn die Ergebnisse einer Mitochondrienbzw. Zellart miteinander verglichen wurden. Er war jedoch für die untersuchten atmenden Objekte mit verschiedenen $\mathrm{QO}_{2}$ nicht gleich $\left(22,5{ }^{\circ} \mathrm{C}\right)$.

Für Herzmuskelsarkosomen (mit der größten $\mathrm{O}_{2}$ Aufnahme/mg Protein) war der KD 90\% am niedrigsten, während er bei niedriger $\mathrm{O}_{2}$-Aufnahme pro mg

* Als KD 90\% haben wir den Druck bezeichnet, bei dem die Geschwindigkeit des $\mathrm{O}_{2}$-Verbrauches $90 \%$ der unter Luft gemessen war.
Protein (also bei Rattenleber-Mitochondrien und Ascites-Tumor-Mitochondrien) am größten war. Es bestand also eine umgekehrte Proportionalität zwischen KD 90\% und der maximalen $\mathrm{O}_{2}$-Aufnahme/ mg Mitochondrien-Protein.

\section{Abhängigkeit des HWD von der Maximal- geschwindigkeit}

Die HWD's wurden durch Anlegen von Tangenten an die registrierten Kurven bestimmt und zur Maximalgeschwindigkeit in Beziehung gesetzt (Abb. 2). Mit zunehmender Maximalgeschwindigkeit (d. h. mit zunehmender Zell- oder Mitochondrienmenge) bleiben die HWD's zunächst konstant, steigen aber bei einer für die jeweiligen Objekte verschiedenen „Grenzkonzentration" von Mitochon-

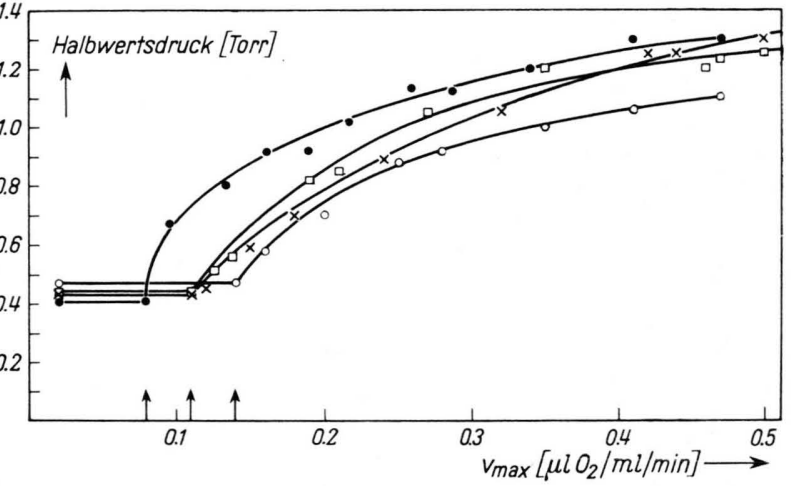

Abb. 2. Abhängigkeit der Halbwertdrucke von der Maximalatmung. $\bullet=$ Ascites-Tumorzellen, $\bigcirc=$ Herzmuskel-Sarkosomen, $\square=$ Rattenleber-Mitochondrien, $\times=$ AscitesttumorMitochondrien. Für jedes Objekt ist die Mittelwertkurve von ca. 80 Meßpunkten eingetragen. Auf der Abszisse ist die jeweilige Grenzmaximalatmung durch Pfeile eingezeichnet.

drien bzw. Zellen, die einer bestimmten „Grenzmaximalatmung“ entspricht, an. Diese „Grenzmaximalatmung " betrug für Tumorzellen $0,06 \mu \mathrm{l} \mathrm{O}_{2} / \mathrm{ml} /$ Min., für Herzmuskel-Sarkosomen $0,16 \mu \mathrm{ll} \mathrm{O}_{2} / \mathrm{ml} /$ Min., für Leber- und Ascites-Tumor-Mitochondrien $0,11 \mu \mathrm{l} \mathrm{O}_{2} / \mathrm{ml} / \mathrm{Min}$. (Diese Werte haben wir für $22,5{ }^{\circ} \mathrm{C}$ gemessen. Mit zunehmender Temperatur wurde die Grenzmaximalatmung vergrößert.) Der HWD erreichte bei den höchsten untersuchten Maximalgeschwindigkeiten von $0,55 \mu \mathrm{l} \mathrm{O} / \mathrm{ml} / \mathrm{Min}$. einen Grenzwert von 1,2 bis 1,3 Torr. Der Tab. 1 liegen die Mittelwerte der HWD's von Geschwindigkeiten von $0,02 \mu \mathrm{l} \mathrm{O} / \mathrm{ml} / \mathrm{Min}$. bis zur jeweiligen „Grenzmaximalgeschwindigkeit" zugrunde. Diese Halbwertdrucke waren um so niedriger, je kleiner die Maximalatmung der untersuchten Objekte war. 


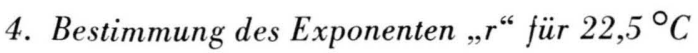 [nach Gl. (1)]}

Es wurden nur solche Druck-Zeit-Kurven für die Berechnung verwendet, deren Maximalgeschwindigkeit unter der „Grenzmaximalatmung“ lagen, d. h. deren HWD mit zunehmender Zellkonzentration nicht erhöht wurde (Abb. 2). Für jede Druck-ZeitKurve wurde für 15 verschiedene Drucke zwischen 0 und 6,5 Torr $\mathrm{O}_{2}$ durch Anlegen von Tangenten an die registrierten Meßkurven die Geschwindigkeit „v" gemessen *.

Trägt man $\log \frac{v}{v_{\max }-v}$ gegen $\log P_{\mathrm{O}_{2}}$ auf (Abb. 3), so liegen die ermittelten Werte auf Geraden, deren Neigung,$r "$ ist. „r" $r$ ist für Sarkosomen, Rattenleber - Mitochondrien, Ascites-Tumor-Mitochondrien und -zellen verschieden groß (Tab. 2). Zwischen 4 und 6 Torr gilt ein Exponent „s“, der wesentlich größer ist als „r“. „s“ war vom Alter der Mitochondrien abhängig. Bei ganz frischen Mitochondrien mit noch gekoppelter Phosphorilierung war,$s "$ größer und der KD niedriger als bei gealterten Mitochondrien. (Der Exponent „r“ wird mit zunehmender Temperatur größer.)

In das Diagramm (Abb. 3) sind die aus den Arbeiten von $\mathrm{W}_{\mathrm{ARBURG}}{ }^{2}$ und $\mathrm{W}_{\text {INZLeR }}{ }^{5}$ entnommenen Werte zum Vergleich eingetragen. Für denjenigen Teil der Kurve, in dem der Exponent „r" gilt, haben wir die Mich a elis - Funktion integriert.

$$
\frac{v_{\max }-v}{v} P_{\mathrm{O}_{2}}^{r}=\frac{K_{\mathrm{r}}}{K_{0}} \quad \frac{K_{\mathrm{r}}}{K_{0}}=K_{\mathrm{m}} \quad r=1,4
$$

durch Auflösen nach $v$ ergibt sich

$$
v=\frac{v_{\max } \cdot P_{\mathrm{O}_{2}}^{r}}{K_{\mathrm{m}}+P_{\mathrm{O}_{2}}^{r}} \quad \frac{\mathrm{d} P \mathrm{O}_{2}}{\mathrm{~d} t}=-v
$$

(mit wachsender Zeit nimmt der Druck ab)

$$
\begin{gathered}
\frac{\mathrm{d} t}{\mathrm{~d} P_{\mathrm{O}_{2}}^{r}}=\left[\frac{K_{\mathrm{m}}}{v_{\max }} \cdot P_{\mathrm{O}_{2}}^{-r}+\frac{1}{v_{\max }}\right] \\
\int_{t_{\mathrm{o}}}^{t_{1}} \mathrm{~d} t=-\int_{P_{\mathrm{o}}}^{P_{1}}\left[\frac{K_{\mathrm{m}}}{v_{\max }} \cdot P_{\mathrm{O}_{2}}^{-r}+\frac{1}{v_{\max }}\right] \mathrm{d} P_{\mathrm{O}_{2}} \\
\int_{0}^{t} \mathrm{~d} t=\frac{1}{v_{\max }} \int_{P}^{P_{\mathrm{o}}}\left[K_{\mathrm{m}} \cdot P_{\mathrm{O}_{2}}^{-r}+1\right] \mathrm{d} P \\
t=\frac{1}{v_{\max }}\left[\left(P^{1-r}-P_{0}^{1-r}\right) \frac{K_{\mathrm{m}}}{1-r}+\left(P_{0}-P\right)\right]
\end{gathered}
$$

* „v“ wurde für jeden Druck und jede Kurve 3-mal bestimmt und gemittelt. Es wurden für jedes untersuchte Objekt etwa 10 Kurven ausgewertet.

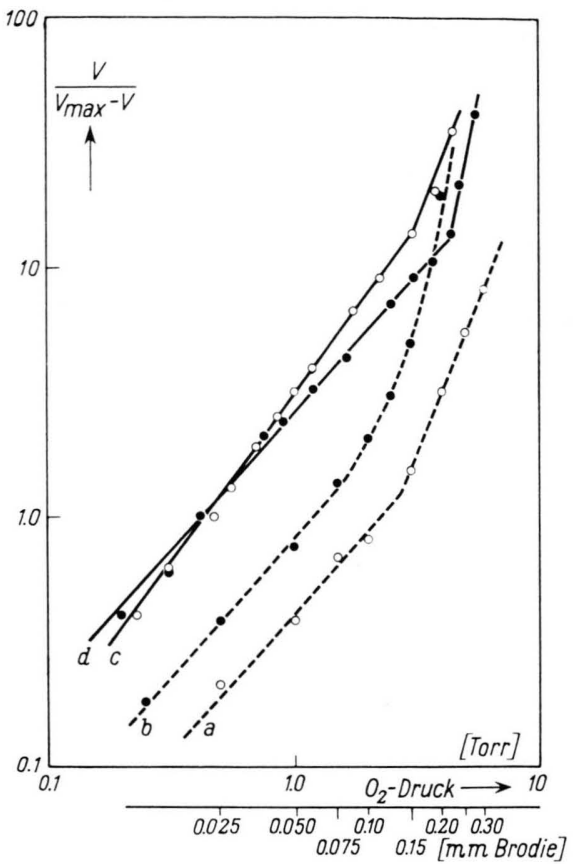

Abb. 3. Bestimmung de̊s Exponenten „ $r$ “ für a) Micrococcus candidans, $10^{\circ} \mathrm{C} 2$, b) Bäckerhefe, $34,3^{\circ} \mathrm{C}^{5}$, c) AscitesTumor-Mitochondrien, $22,5^{\circ} \mathrm{C}$, d) Herzmuskel-Sarkosomen, $22,5^{\circ} \mathrm{C}$. Für Herzmuskel-Sarkosomen und Tumorzell-Mitochondrien gilt,$r^{\prime \prime}$ bis $93 \%$ der Maximalatmung. Für die Funktion a ist der $\mathrm{O}_{2}$-Druck in mm Brodie angegeben.

$K_{\mathrm{r}}=$ Geschwindigkeitskonstante der Reduktion des $\mathrm{O}_{2}$-übertragenden Fermentes, $K_{0}=$ Geschwindigkeitskonstante der Oxydation des $\mathrm{O}_{2}$-übertragenden Fermentes, $v=$ Geschwindigkeit der $\mathrm{O}_{2}$-Aufnahme bei $\mathrm{O}_{2}$-Druck $P_{\mathrm{O}_{0}}, v_{\max }=$ Geschwindigkeit der $\mathrm{O}_{2}$ Aufnahme unter Luft, $t=$ Zeit in Minuten, $P_{0}=$ Partialdruck von $\mathrm{O}_{2}$ zur Zeit $t_{0}, P=$ Partialdruck von $\mathrm{O}_{2}$ zur Zeit $t$.

In Tab. 3 ist die mathematische Deutung der $\mathrm{O}_{2}$ Aufnahme für verschiedene atmende Partikel zusammengestellt.

Die durchzogenen Linien der Kurven in Abb. 4 sind nach Originalregistrierung des $\mathrm{O}_{2}$-Verbrauches von Herzmuskel-Sarkosomen in Abhängigkeit vom $\mathrm{O}_{2}$-Druck gezeichnet. Die eingetragenen Punkte wurden nach Gl. (2) berechnet. Für Kurve a stimmen die errechneten Werte gut mit dem Verlauf der Atmung überein. Das war der Fall, wenn wir eine Maximalgeschwindigkeit wählten, die unter der ermittelten Grenzmaximalatmung des HWD lag $(0,02$ bis $0,16 \mu \mathrm{l} / \mathrm{ml} / \mathrm{Min} .22,5^{\circ} \mathrm{C}$ ). Steigerten wir die Maximalgeschwindigkeit durch Erhöhung der Sarkosomen-Konzentration über die für unser Meßsystem 


\begin{tabular}{|c|c|c|c|c|}
\hline Objekt & $\begin{array}{c}\text { maximaler } \mathrm{O}_{2} \text {-Verbrauch } \\
\text { pro } \mathrm{mg} \mathrm{E} / 2 \mathrm{ml} \text { Ansatz } \\
{[\mu \mathrm{l}]}\end{array}$ & Exponent,$r^{\prime}$ & $\begin{array}{l}\text { Exponent }, r^{\prime \prime} \\
\text { gilt bis } \% \text { der } \\
\text { Maximalatmung }\end{array}$ & $\begin{array}{c}\text { Temperatur } \\
{\left[{ }^{\circ} \mathrm{C}\right]}\end{array}$ \\
\hline Bäckerhefe 5 & & 1,10 & 55 & 34,2 \\
\hline Microc. candidans ${ }^{2}$ & & 1,10 & 55 & 10,0 \\
\hline Ascites-Tumorzellen & & 1,32 & 93 & 22,5 \\
\hline Clorella $^{19}$ & & 1,40 & - & 20,0 \\
\hline Leber-Mitochondrien ${ }^{3}$ & & 1,40 & - & - \\
\hline theoretisch berechnet ${ }^{11}$ & & 1,33 & - & - \\
\hline Ascites-Tumor-Mitochondrien & 0,185 & 1,07 & 93 & 22,5 \\
\hline Leber-Mitochondrien & 0,240 & 1,23 & 93 & 22,5 \\
\hline Herzmuskel-Sarkomen & 0,300 & 1,40 & $\begin{array}{r}93 \\
\ldots\end{array}$ & 22,5 \\
\hline
\end{tabular}

Tab. 2. Die Größe des Exponenten „r“ der Funktion (1) für verschiedene atmende Partikel.

angegebene Grenzmaximalatmung (Kurve b), so waren die Bedingungen für die Gl. (2) nicht mehr erfüllt. Gleichzeitig steigt dann, wie aus Abb. 2 ersichtlich ist, der HWD an. Die errechneten Punkte lagen dann unter der registrierten Atmungskurve. Das Gleiche galt auch für Leber- und Ascites-TumorMitochondrien. Der KD 90\% ist hingegen bei beiden Kurven gleich groß. Er änderte sich erst bei Maximalgeschwindigkeiten über $0,5 \mu \mathrm{l} / \mathrm{ml} /$ Minute.

5. Die Aktivierungsenergie für die $\mathrm{O}_{2}$-Aufnahme wurde für Herzmuskelsarkosomen nach der A r -
rhenius-Gleichung berechnet. Der $\log v$ (Atmungsgeschwindigkeit) für verschiedene Temperaturen wurde gegen $1 / T$ aufgetragen. Aus der Neigung der Geraden wurde eine Aktivierungsenergie von $16440 \mathrm{cal}$ berechnet.

\section{Diskussion}

\section{Bemerkungen zur Methode}

Als limitierender Faktor muß bei unseren Versuchen die Erhöhung der Halbwertdrucke und auch

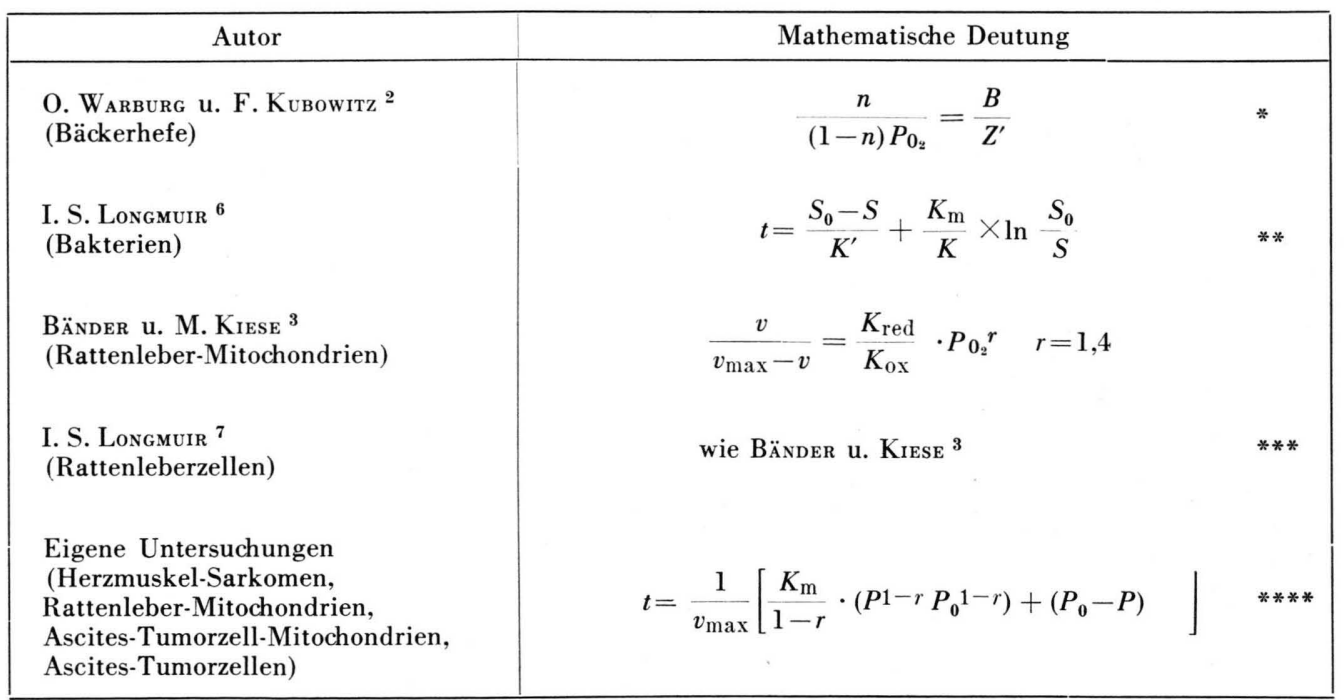

Tab. 3. Mathematische Deutung des Verlaufes der Atmung (im Bereich des KD) in Abhängigkeit vom $\mathrm{P}_{0_{2}}$.

* $B=$ Geschwindigkeitskonstante der Oxydation des $\mathrm{Fe}, \quad Z^{\prime}=$ Geschwindigkeitskonstante der Reduktion des Fe, $n=A / A_{0}, A=V$ (Geschwindigkeit der Atmung bei $\left.P_{0_{2}}\right) ; A_{0}=v_{\max }$ (Geschwindigkeit der Atmung unter Luft). Setzt man in die Formel von $\mathrm{W}_{\mathrm{ARBU}} \mathrm{u}$. Kubowitz ${ }^{2}$ für $n=v / v_{\max }$ ein, so erhält man durch Umformung $\frac{v}{\left(v_{\max }-v\right) P \mathrm{O}_{2}}=\frac{B}{Z^{\prime}} \operatorname{die}$ Funktion von B̈̈nder u. Kiese, die sich von der W $\mathrm{W}_{\text {ARBurg schen }}{ }^{2}$ durch $P_{0_{2}}{ }^{1,4}$ unterscheidet. ${ }^{*}$ Nach Longmuir ${ }^{6}$ folgt die Atmung von Bakterien dieser Formel, erhalten durch Integration der $\mathrm{M}$ i c h a e li s - Funktion für den Exponenten 1 für $P_{0_{2}}$. ${ }^{* * *}$ Bestätigt für einen bestimmten Bereich die von Bänder u. Kiese ${ }^{3}$ gefundene Funktion mit dem Exponenten $r=1,4$ für Rattenleberzellen. *** Die Druckzeitkurven der Atmung folgen in einem bestimmten Bereich der angegebenen Funktion. Erhalten durch Integration der M i c h a e lis - Funktion für den Exponenten $r=\neq$ von $^{*}$. * $\neq=$ verschieden. 


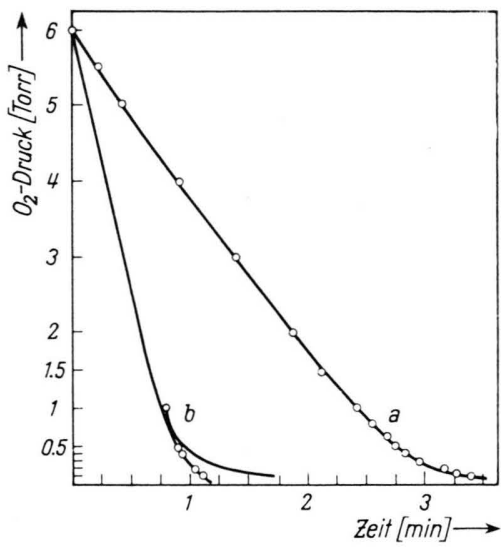

Abb. 4. a) Originalkurve der Atmung von Herzmuskel-Sarkosomen in Abhängigkeit vom $\mathrm{O}_{2}$-Druck - durchgezogene Linie. Die Punkte sind errechnete Werte nach Gl. (2) $\left(v_{\max }=0,083 \mu \mathrm{l} \quad \mathrm{O}_{2} / \mathrm{ml} / \mathrm{Min} ., \quad T=22,5^{\circ} \mathrm{C}\right) . \quad$ b) Originalmeßkurve für eine Maximalgeschwindigkeit oberhalb der „Grenzmaximalatmung“. Die errechneten Punkte liegen hier nicht mehr auf der Meßkurve $\left(v_{\max }=0,26 \mu \mathrm{l} \mathrm{O} / \mathrm{ml} / \mathrm{Min}\right.$., $\left.T=22,5^{\circ} \mathrm{C}\right)$. Die Punkte sind nach Gl. $(2)$ mit $r=(1,4)$ und $s=2,0$ errechnet.

der Kritischen Drucke bei gleichen Objekten oberhalb einer Grenzmaximalatmung angesehen werden.

Nach der Beziehung $i_{\mathrm{g}}=i_{\mathrm{g}_{0}} \cdot e \frac{-D \cdot q \cdot t}{\delta \cdot V}$ ist der Dif-

fusionsgrenzstrom $\left(i_{g}\right)$ bei möglichst schneller und konstanter Rührung und damit konstant bleibender Diffusionsschicht $(\delta)$ der $\mathrm{O}_{2}$-Konzentration im Ansatz proportional. Es ist jedoch zu beachten, daß in der Diffusionsgrenzschicht selbst $\mathrm{O}_{2}$-verbrauchende Vorgänge stattfinden. Nach WinkelmanN ${ }^{15}$ können dann noch Änderungen der $\mathrm{O}_{2}$-Spannung, deren Halbwertszeiten nicht unter der Größe von einigen Sek. liegen, hinreichend genau gemessen werden. Für unsere Ergebnisse erhebt sich nun die Frage, warum der HWD und auch der KD 90\% oberhalb einer Grenzmaximalatmung mit steigender Mitochondrien-Konzentration bzw. Maximalatmung zunimmt. Folgende Ursachen könnten für dieses Verhalten verantwortlich gemacht werden:

a) Beimischungen von Hämoglobin bzw. Myoglobin könnten durch Freisetzung des gebundenen Sauerstoffes bei abnehmendem $\mathrm{O}_{2}$-Druck in der Meßlösung für diesen Störeffekt verantwortlich gemacht werden. Dagegen sprechen jedoch die folgenden Argumente:

15 D. Winkelmann, Ber. Bunsenges. physik. Chem. 59, 895 [1955].

16 B.Chance u. B. Hess, J. biol. Chemistry 234, 2404 [1959].
Ascites-Tumor-Mitochondrien können frei von Hämoglobin präpariert werden ${ }^{16}$. Trotzdem ergab sich auch hier eine Abhängigkeit des HWD und KD 90 Prozent. Bei Hämoglobinzusatz zu den Versuchsansätzen kamen Meßkurven zustande, die mit unseren nicht vergleichbar waren. Aus theoretischen Überlegungen geht hervor, daß sich bei steigender Hämoglobin-Konzentration im Versuchsansatz zuerst der KD 90\% und erst in zweiter Linie der HWD ändern müßte. In unseren Versuchen wurde jedoch bei steigender Mitochondrien-Konzentration (bzw. Maximalatmung) zuerst der HWD beeinflußt.

b) Sowohl Warburg, Meyerhof und Schulz ${ }^{16}$, Elliott und Henry ${ }^{17}$ als auch Winzler ${ }^{5}$, weisen darauf hin, daß ihre Messungen nur dann als reell anzusehen sind, wenn bei zunehmender Verdünnung der atmenden Partikel, nicht aber wie Kempner ${ }^{18}$ ausführt, durch Erhöhung der Schüttelgeschwindigkeit das Verhältnis $v / v_{\max }$ unverändert bleibt. Da bei unseren polarographischen Messungen kein Gasraum über dem Suspensionsmedium ist, kann die Diffusion vom Gasraum in das Medium ausgeschlossen werden. Die von uns gemessene Grenzmaximalatmung könnte nur im Verhalten der atmenden Partikel selbst zu erklären sein. In Anlehnung an die von Meyerhof ${ }^{16}$ gegebene Beziehung $R^{\prime}=\sqrt{\frac{6 \cdot c_{0} \cdot D}{A}}$ kann die Diffusion nicht zum limitierenden Faktor werden, wenn $R^{\prime}$ wesentlich größer ist als $R$. Geht man von der Annahme aus, da $\beta$ mit zunehmender Konzentration der Partikel auch ihre Aggregation zunimmt, so kann man berechnen, daß der HWD mit zunehmender Konzentration der Mitochondrien eher beeinflußt wird, als der KD 90 Prozent. Dafür könnte die Zunahme des Diffusionsgefälles von der Oberfläche zum Mittelpunkt der aggregierten atmenden Partikel verantwortlich gemacht werden. Diesen theoretischen Überlegungen entsprachen unsere experimentellen Ergebnisse.

\section{Der kritische Sauerstoffdruck} (K D )

Der KD und auch der KD 90\% war für verschiedene Objekte bis zu $0,5 \mu \mathrm{l} \mathrm{O}_{2} / \mathrm{ml} / \mathrm{Min}$. (Grenzmaximalatmung des KD 90\%) dem $Q_{\mathrm{O}_{2}} / \mathrm{mg}$ Protein $u m$ gekehrt proportional. Für dieses Verhalten gibt es

17 K. A. C. Elliott and M. Henry, J. biol. Chemistry 163, 351 [1946].

18 W. Kempner, Cold Spring Harbor Sympos. quantitat. Biol. VII, 269 [1939]. 
folgende Erklärung: Chance ${ }^{11}$ konnte durch theoretische Berechnungen und Experimente zeigen, daß die terminale Oxydase bereits teilweise reduziert sein kann, ohne daß die Atmungsgeschwindigkeit, gemessen an der Fumaratproduktion bzw. der $\mathrm{O}_{2}$ Aufnahme, verändert ist. Der Reduktionsgrad blieb mit abnehmendem $\mathrm{O}_{2}$-Druck konstant, solange die Atmungsgeschwindigkeit maximal war. Vom KD an nahm die Atmung ab und näherte sich dem Nullwert. Erst wenn die Atmung den KD erreicht hatte, wurde die terminale Oxydase sehr schnell reduziert. Eine Erklärung für diese plötzliche Reduktion der Oxydase ohne übereinstimmende Änderung der Atmung wäre das Vorhandensein eines Überschusses an terminaler Oxydase in der Atemkette der Säuger ${ }^{11}$. Nur so kann sich ihr Redox-Grad erheblich ändern, ohne daß gleichzeitig eine meßbare Änderung der Atmung erfolgt. Dieser „cushioning effect“ (Polstereffekt) kann eine Erklärung dafür sein, daß je nach der Größe des Überschusses an terminaler Oxydase bei atmenden Partikeln, verschiedene kritische Drucke gemessen werden. Chance ${ }^{11}$ weist auf die variable Affinität der Atemkette zum Sauerstoff bei verschiedenem metabolischen Status der Mitochondrien hin. Je mehr aktivierte Enzyme der Atemkette bei den verschiedenen atmenden Partikeln verfügbar sind und je größer der Überschuß an terminaler Oxydase ist, um so größer ist der $Q_{\mathrm{O}_{2}} / \mathrm{mg}$ Protein und der "cushioning effect". Um so niedriger wird dann der $\mathrm{O}_{2}$-Druck, bei dem die Atmung von der Maximalatmung abweicht. Eine Abnahme des KD mit zunehmender $Q_{\mathrm{O}_{2}}$ fand David C. White ${ }^{9}$ auch durch Messung der Atmung an enzymarm und enzymreich gezüchteten Kulturen von B. haemophilus parainfluenzae. Wenn wir durch Erhöhung der Temperatur den Umsatz steigerten, nahm der KD ab.

Wenn die Mitochondrien verschiedener Organe einen von der Enzymaktivität und vom metabolischen Status abhängigen KD haben, so könnten unsere Messungen zu der Erklärung beitragen, daß unter zunehmendem $\mathrm{O}_{2}$-Mangel des Organismus physiologische Funktionen in einer bestimmten vom KD abhängigen Reihenfolge ausfallen.

\section{Der Halbwertdruck HWD (, $\left.K_{\mathrm{m}}{ }{ }^{\prime}\right)$}

Die Vermutung von $W_{\text {ARBURG }}{ }^{2,19}$, daß bei Zellen der HWD um so kleiner ist, je kleiner ihr $\mathrm{O}_{2}$-Verbrauch ist, trifft nach unseren Messungen auch für Mitochondrien zu. Mit abnehmenden $Q_{\mathrm{O}_{2}}$ wird der
HWD kleiner und ist bei Tumor-Mitochondrien am niedrigsten. Diese Abhängigkeit gilt nur für einen Vergleich der Halbwertdrucke an verschiedenen Objekten bis zur "Grenzmaximalatmung“ des HWD. Auf eine Abhängigkeit der Halbwertdrucke vom $Q_{\mathrm{O}_{2}}$ weisen auch Meyerhof und $\mathrm{S}_{\mathrm{CHulz}}{ }^{16} \mathrm{hin}$, die den $\mathrm{O}_{2}$-Verbrauch von Bakt. azotobakt. chroococcum durch Alterung der Kultur variierten und manometrisch gemessen haben. $\mathrm{Da} ß$ eine solche Abhängigkeit des HWD möglich ist, konnten Chance, Higgins, Holmers und BARR ${ }^{11}$ durch theoretische Berechnungen mit Hilfe eines Analogrechners zeigen. Die Untersucher haben in einer angenommenen Folge von 3 Enzymen die Abhängigkeit des HWD ( „K $\left.K_{\mathrm{m}}{ }^{\circ}\right)$ von der Konzentration des mittleren Enzyms berechnet. Danach hat die Erhöhung der Enzymkonzentration bei gleichbleibend hoher Substratkonzentration eine Zunahme des HWD ( „ $K_{\mathrm{m}}$ “) zur Folge. Nach diesen theoretischen Berechnungen kann die Zunahme der HWD's bei unseren Untersuchungen mit steigen$\operatorname{dem} Q_{\mathrm{O}_{2}} / \mathrm{mg}$ Protein bei den einzelnen Objekten durch verschiedene Enzymkonzentrationen und deren Verhältnis innerhalb der Sequenz der Atemkette zueinander bedingt sein. Diese Ergebnisse an Mitochondrien stehen allerdings im Gegensatz zu denen, die David C. $\mathrm{W}_{\text {HITE }}{ }^{9}$ bei Bakterien gefunden hat. Dieser Autor fand mit zunehmenden $Q_{\mathrm{O}_{2}}$ eine Abnahme der HWD's.

Ein Vergleich der kritischen- und Halbwertdrucke von intakten Tumorzellen und Tumorzell-Mitochondrien zeigt, daß sowohl der KD als auch der HWD für intakte Zellen und isolierte Mitochondrien etwa gleich ist. Daß der Diffusionsweg des Sauerstoffs durch das Zellplasma weder den KD noch den HWD erhöht, konnte $\mathrm{W}_{\text {INZLER }}{ }^{5}$ für Bäckerhefe mit Hilfe der CO-Hemmung ausschließen. Ebenso fand LongMUIR $^{7}$, daß KD und HWD für Rattenleberzellen und Mitochondrien etwa gleich sind. Diese Verhältnisse scheinen auf Bakterien nicht übertragbar zu sein. LoNGMUIR $^{6}$ fand bei Bakterien mit zunehmendem Zelldurchmesser eine Zunahme der Halbwertdrucke und führte diese auf die Diffusion von Sauerstoff durch das Plasma zurück.

$$
\begin{array}{r}
\text { 4. Der Exponent "r" der Funktion } \\
\text { vgl. S. } 240
\end{array}
$$

Unsere Messungen zeigen, daß der von BäNDER und KIEsE ${ }^{3}$, für Rattenleber-Mitochondrien gefundene Exponent der Funktion (1) nicht allgemein zutrifft. Der Exponent „ $r^{\text {“ }}$ war bei unseren Versu- 
chen vom $Q_{\mathrm{O}_{2}}$ und von der Temperatur der verschiedenen untersuchten Objekte abhängig. Wir haben in das Diagramm (Abb. 3) die von W ARBurg $^{2}$ und $\mathrm{W}_{\text {INZLER }}{ }^{5}$ gemessenen Werte zum Vergleich eingetragen. Auch bei den Ergebnissen dieser Autoren war der Exponent für einige atmende Objekte verschieden von 1,4 . Nur bei Chlorella ${ }^{19}$ ergab sich ebenso wie bei Herzmuskelsarkosomen $\left(22,5{ }^{\circ} \mathrm{C}\right)$ ein Exponent von 1,4. Für Bakterien folgte die Funktion den Exponenten $1^{6}$. Für Warmblüterzellen und Mitochondrien konnten wir niemals einen Exponenten von 1 finden. Wir haben aus diesem Grunde die Michaelis-Funktion für einen Exponenten ${ } r^{“}=$ verschieden von 1 integriert. Setzt man in die so erhaltene Formel (2) die gefundenen Meßwerte ein, so liegen die errechneten Punkte in guter Näherung auf den registrierten Meßkurven, sofern die Grenzmaximalatmung nicht überschritten wird (Abb. 4). Longmuir $^{7}$ fand bei Rattenleberzellen ebenfalls einen Exponenten von 1,4. Aus Abb. 3 und Tab. 2 geht hervor, daß der Bereich, in dem , $r$ “ gilt, bei den einzelnen untersuchten Objekten verschieden ist. Dieses Verhalten ist vielleicht dadurch

19 O. Warburg u. G. Krippahl, G. Thieme-Verlag Stuttgart, S. 427,1962 . zu erklären, daß bei niedrigen $\mathrm{O}_{2}$-Drucken außer dem Succinoxydase-System noch eine andere Komponente limitierend ist.

Wie kann man sich das Zustandekommen des für eine chemische Reaktion ungewöhnlichen Exponenten ${ }^{\prime \prime}$ " und dessen Abhängigkeit vom $Q_{\mathrm{O}_{2}}$ erklären? Die mathematische Theorie der Reaktion der Cytochromoxydase mit $\mathrm{O}_{2}$ zeigt, daß die Reaktion in bezug auf Sauerstoff 1. Ordnung sein kann ${ }^{20}$. Die Reduktion des $\mathrm{O}_{2}$ erfolgt schrittweise über freie Radikale. Die chemischen Vorgänge an der terminalen Oxydase und die der anderen Enzyme der Atemkette lassen sich durch nichtlineare Differentialgleichungen beschreiben. Chance, Holmes, Higgins und $\mathrm{B}_{\mathrm{ARR}}{ }^{11}$ haben diese Gleichungen mit Hilfe des Analogrechners gelöst und geben einen berechneten Exponenten ${ }^{\prime \prime}{ }^{\prime \prime}=1,33$ an. (Den gleichen Exponenten haben wir bei Versuchen mit Ascitestumorzellen bei $22,5{ }^{\circ} \mathrm{C}$ gefunden.) „ $K_{\mathrm{m}}$ “ bzw. der HWD sind nach den theoretischen Berechnungen von Chance ${ }^{11}$ von dem Verhältnis der Enzymkonzentrationen abhängig. Es ist daher anzunehmen, daß auch „r" eine solche Abhängigkeit zeigt.

20 E. King u. Chuan Pu Lee, Biochim. biophysica Acta [Amsterdam] 37, 243 [1960]. 